\title{
Treating oligohydramnios with extract of Salvia miltiorrhiza: A randomized control trial
}

\author{
Hong-nü Chu \\ Mei-juan Shen \\ Affiliated Hospital, School of Medicine, \\ Hangzhou Normal University, 3100 I6, \\ Hangzhou, China
}

\begin{abstract}
Objective: To determine whether purified herbal extract of Salvia miltiorrhiza can improve the amniotic fluid volume in pre-term oligohydramnios by improving uteroplacental circulation.

Methods: Forty-three pregnant women with oligohydramnios received a daily intravenous dose of $30 \mathrm{~mL}$ of salvia extract mixed with $5 \%$ glucose $500 \mathrm{~mL}$. A control group of 41 women received daily $5 \%$ glucose $500 \mathrm{~mL}$ only. The amniotic fluid index (AFI) was assessed at least twice a week by ultrasonographists who were blinded to the treatment. Both women and fetuses were monitored closely. The change in AFI was calculated and compared by paired $t$ test within and between groups. The revised recommendations for improving the quality of reports of parallel group randomized trials were used.

Results: After a mean of $7.2 \pm 2.7$ days' therapy, ranging from 3 to 18 days, the AFI increased significantly from a mean of $4.9 \pm 2.3 \mathrm{~cm}$ to a mean of $7.12 \pm 2.36 \mathrm{~cm}$, by a mean of AFI $0.18 \pm$ $0.06 \mathrm{~cm} /$ day (paired $t=3.62, \mathrm{p}<0.005$ ). In the control group, the AFI increased from a mean of $5.1 \pm 2.4 \mathrm{~cm}$ to a mean of $5.5 \pm 3.1 \mathrm{~cm}$ after a mean of $6.1 \pm 3.3$ days' treatment, ranging from 4 to 15 days. The effect of salvia treatment on AFI in the salvia group was significantly greater than in the control group $(\mathrm{p}<0.001)$. No side effects were observed in treated patients.

Conclusion: Salvia miltiorrhiza is an effective Chinese medicine for the treatment of oligohydramnios.
\end{abstract}

Keywords: Salvia miltiorrhiza, therapy, oligohydramnios, amniotic fluid index

\section{Introduction}

Oligohydramnios, defined as an amniotic fluid index (AFI) of less than the 5 th percentile (at term $\leq 5 \mathrm{~cm}$, or at pre-term $\leq 8.0 \mathrm{~cm}$ ), has an incidence of $8.5 \%$ to $15.5 \%$ (Rutherford et al 1987; Sarno et al 1989; Moor et al 1990), and is associated with an increased risk of umbilical cord occlusion, fetal distress in labor, operative deliveries, and stillbirth at term. However, oligohydramnios observed in the second trimester or the early third trimester is particularly ominous, with perinatal mortality rates approaching $80 \%-90 \%$ (Baess et al 1984; Mercwe et al 1986). When amniotic fluid is absent, the perinatal loss rate increases to $90 \%$ (Moore et al 1989). As shown earlier, oligohydramnios is a result of a decrease in fetal urine production and excretion with intact membranes and without fetal renal anomalies. The cause of the decrease in fetal urine production and excretion is chronic hypoxia because of placental dysfunction, resulting in shunting of fetal blood flow from the kidneys and a decrease in glomerular filtration rate. Therefore, oligohydramnios is usually accompanied by fetal growth restriction.

An effective medical therapy for oligohydramnios is very important for the fetus to grow normally to near term. Though some treatment modalities such as transabdominal amniocentesis and maternal hydration have been suggested, none work well or resolve the primary cause.

In China, salvia, a kind of traditional Chinese herbal medicine extracted from Salvia miltiorrhiza, has been used to treat cardiovascular disease and promote microcirculation 
in microthrombosis for several decades (Zhang et al 2005). In this study, we used salvia to improve maternal blood circulation and enhance placental function, and observed its effect on amniotic fluid volume.

\section{Methods}

The study protocol was approved by the local Institute Review Board and the hospital ethics committee. The purified Salvia miltiorrhiza extract (PSME) was obtained from ZDQCB Corporation Ltd (Zhengjiang zhengda Qingchunbao Yao Ye, Zhengjiang, China, Batch No. 0503087, Z33020177).

After the patients had provided written informed consent, they were admitted to hospital and randomly assigned to the study group or the control group by computer at the admission station. The reduced amniotic fluid volume was assessed by the four-quadrant technique, as described previously, and expressed as centimeters (AFI less than 5th percentile) (Rutherford et al 1987). Ultrasonography was performed for fetal anatomic study and fetal weight estimation. AFI was confirmed by a second examiner using the same color Doppler 3-Dimension ultrasonography machine as the first examiner. Patients who were diagnosed with fetal anomalities by ultrosonography (eg, cardiac anomalies, renal cysts), or who were diagnosed with diabetes, were not enrolled in this study.

Maternal blood samples were drawn for routine baseline laboratory examination, including hemoglobin, hematocrit, platelets, and fibrinogen concentration, for emergency surgery delivery in case of ineffective treatment. Patients were divided into 2 groups: one group of 43 received a daily intravenous injection of $30 \mathrm{~mL}$ salvia extract, provided from inter medicine department, mixed with $5 \%$ glucose $500 \mathrm{~mL}$ over 4-6 hours; a control group 41 received conventional treatment of $5 \%$ glucose $500 \mathrm{~mL}$ only. In addition, $10 \mathrm{mg}$ of dexamethasone was given once a day for 3 days to those patients in both groups at less than 34 weeks of gestation, to promote fetal lung maturity, in case the treatment was ineffective and the pregnancy terminated. The AFI was reassessed at least twice a week by an ultrasonographist who was blinded to the treatment. If the AFI increased to normal levels, the patient was discharged and followed up at clinics until to delivery. If the AFI remained in the abnormal range, the patient treatment was continued until the fetus was viable, or until the pregnancy terminated due to unviable fetus $(<24$ gestational weeks). If the AFI decreased sharply, so that fetal life was threatened, or labor began, the pregnancy was concluded by caesarian section. During therapy, fetal wellbeing was monitored by: (1) fetal heart rate with a Doppler auscultator every 2 hours; (2) fetal body movement counting by the mother 3 times a day for 1 hour each; (3) nonstress test, fetal electrocardiogram, vibro-acoustic stimulation test, and biophysical profile were performed at least twice a week.

If any evidence of harmful effects to the mother or fetus were found during treatment, the therapy was stopped and normal management was used. The study ceased when sufficient data had been collected for statistical analysis. The revised recommendations for improving the quality of reports of parallel group randomized trials were used (Moher et al 2001).

\section{Data analysis}

All values were expressed as mean \pm SEM. Changes in AFI were analyzed by paired $t$ test. Statistical significance was set at $\mathrm{p}<0.05$. Assumptions made at the start of the study were: (1) that the estimated variance of the paired differences was $25 \%$; (2) the certainty that the difference detected was at the 0.05 level and was not because of chance (eg, significance level or $\alpha=0.05)$; (3) the test power is $0.8(\beta=0.80)$. Therefore, 16 patients were needed in this study for paired $t$ test; 32 patients were needed for two samples comparison. The government or patients paid for all costs depending on their medicare policy.

\section{Results}

Forty-three pregnant women were enrolled in the salvia proto$\mathrm{col}$, and 41 patients in the control group. Mean age of women was $28.5 \pm 2.3$ years (range 21-39 years) (Table 1). Most patients $(80 / 84=91 \%)$ were primigravida. Mean gestational age was $32.0 \pm 3.5$ weeks (range 23-36 weeks). At admission, mean AFI was $4.9 \pm 2.3 \mathrm{~cm}$ (range $1.0-7.9 \mathrm{~cm}$ ).

Table I The clinical characteristics and the results of treatment with salvia and the control (mean \pm SD or Cl 95\%)

\begin{tabular}{llll}
\hline & Salvia group & Control group & $\mathbf{P}$ \\
\hline Maternal ages (year old) & $28.3 \pm 2.2$ & $28.5 \pm 2.3$ & $\mathrm{NS}$ \\
& {$[23.9,32.7]$} & {$[23.9,33.1]$} & \\
Gestational age (weeks) & $32.0 \pm 3.5$ & $31.1 \pm 3.7$ & $\mathrm{NS}$ \\
& {$[25.0,39.0]$} & {$[23.7,38.5]$} & \\
Amniotic fluid index (cm) & & & \\
Pretreatment & $4.9 \pm 2.3^{*}$ & $5.1 \pm 2.1$ & $\mathrm{NS}$ \\
& {$[1.3,10.5]$} & {$[0.9,9.3]$} & \\
Post-treatment & $7.1 \pm 2.4$ & $5.5 \pm 3.1$ & $\mathrm{~S}$ \\
& {$[2.3,11.9]$} & {$[0,11.7]$} & \\
Duration of therapy (days) & $7.2 \pm 2.7$ & $6.9 \pm 3.8$ & $\mathrm{NS}$ \\
& {$[1.8,12.6]$} & {$[0.14 .5]$} & \\
Increased velocity (cm/day) & $0.18 \pm 0.06$ & $0.05 \pm 0.02$ & $\mathrm{~S}$ \\
& {$[0.02,0.30]$} & {$[0.01,0.09]$} & \\
Absolute efficacy rate & $70(30 / 43) \%$ & $24.4(10 / 44) \%$ & $\mathrm{~S}$ \\
Relative efficacy rate & $84(36 / 43) \%$ & $36.6(15 / 4 I) \%$ & $\mathrm{~S}$ \\
\hline
\end{tabular}

Notes: In the salvia group, the amniotic fluid index was significantly increased posttreatment compared with pretreatment (paired $t=3.62, \mathrm{p}<0.005$ ). 
After a mean of $7.2 \pm 2.7$ days' therapy, ranging from 3 to 18 days, the AFI increased significantly to a mean of $7.1 \pm 2.4$ $\mathrm{cm}$, ranging from 0 to $10.2 \mathrm{~cm}$ (paired $t=3.62, \mathrm{p}<0.005$ ). The AFI increased by a mean of $0.18 \pm 0.06 \mathrm{~cm} /$ day. Thirteen patients failed to respond to therapy. Among these patients, 6 showed no significant increase in AFI, but maintained the pretreatment level. Six patients showed a decrease in AFI after treatment, and were delivered by caesarean section. They had viable infants. One of the 13 patients had an inviable fetus and determined of pregnancy at 23 weeks of gestation, due to an extremely low fluid level. The absolute (increased fluid) effective rate of salvia was approximately $70 \%$. The relative (though AFI did not increase, it was not easy to maintain pretreatment level due to insufficient placenta function) effective rate of salvia was $84 \%$. The patients showed no side effects. There was no drop out due to adverse effects during the clinical trial. The details of the control group are set out in Table 1 .

\section{Discussion}

The results of this clinical trial demonstrate that administration of salvia can increase the level of amniotic fluid in general, and improve the fetal survival environment without maternal side effects. The salvia treatment of oligohydramnios has never been reported in English. In China, and other countries, salvia has been used as therapy for cardiovascular disease, chronic renal insufficiency, and intrahepatic cholestasis for at least 30 years (Fugh-Berman 2000; Kuang et al 2005; Adams 2006). According to traditional Chinese medicine theory, the decrease in urine volume can be attributed to insufficient blood flow to the kidneys, due to reduced blood volume and blood stasis. Urine volume is an important resource of fluids during pregnancy. Many animal experiments and clinical phase I to phase III trials have demonstrated that salvia can activate blood circulation and remove blood stasis. Salvia includes many active compounds, including magnesium lithospermate A, B, C, D, E, F, G, H, I. Previously published research has shown approximately 25 kinds of effects of these compounds, including magnesium lithospermate, dipotassium lithospermate, sodium rosmarinate and potassium danshensu, dipotassium isolithospermate $\mathrm{B}$ and magnesium salvianolate $\mathrm{G}$. The most important of these is magnesium lithospermate $\mathrm{B}$, a tetramer of caffeic acid, which is a most powerful efficacious compound. Figure 1 shows chromatograms of standard mixtures (Xu et al 2007). The product here is similar to the standard with $95.0 \%$ to $99.9 \%$ and good reproducibility and stability ( $\mathrm{Xu}$ et al 2007). They can ameliorate renal ischemia and improve blood circulation and microcirculation in the kidney, thereby

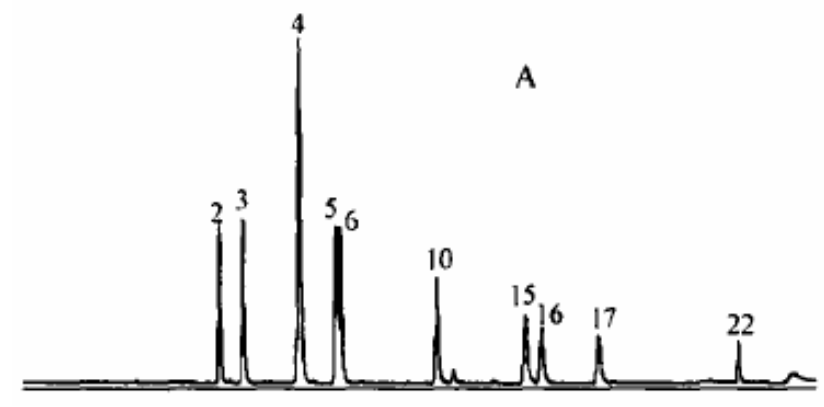

Figure I Liquid chromatography - mass spectrometry: Chromatograms of standard mixtures (Xu et al 2007).

enhancing the glomerular filtration rate and increasing urine (Yokozawa et al 1989; Sun et al 2005).

In pregnant women with oligohydramnios, plasma viscosity and coagulation parameters were significantly higher before onset (Battaglia et al 1995). In the oligohydramnios placenta, there exist platelet and fibrin aggregates and thrombosis of the fetal vessels, suggesting that a hypercoagulative state and hyperfibrinolysis might affect the microcirculation and result in microthrombis, obliteration or sclerosis in the small arteries and arteries of the tertiary villi of the placental cotyledon (Redline et al 1995). Salvia treatment can regulate capillary tension and improve hemorrheological properties, by affecting the activity of fibrinolysis and suppressing platelet aggregation, reducing blood viscosity and improving microcirculation. In this way, the fetus can gain sufficient blood from the placenta and excrete urine normally, leading to a gradual increase in amniotic fluid. As we know, fetal urine production is the major source of amniotic fluid at the second and third trimesters. However, there is no effective treatment for a low fluid level, if the placenta has been severely changed pathologically, such as by extreme fibrin formation, calcification, infarction or embolism.

In conclusion, salvia therapy oligohydramnios is a primary study. The potential benefits and its detailed mechanisms need further in-depth study at multiple centers, based on large samples and case-controlled design. This treatment is an exciting finding for clinical practice.

\section{Disclosure}

The authors declare there are no conflicts of interest.

\section{References}

Adams JD, Wang R, Yang J, et al. 2006. Preclinical and clinical examinations of Salvia miltiorrhiza and its tanshinones in ischemic conditions. Chin Med, 1:663-94.

Barss VA, Benacerraf BR, Frigoletto FD. 1984. Second trimester oligohydramnios, a predictor of poor fetal outcome. Obstet Gynecol, 64:608-13. 
Battaglia C, Artini PG, Ballestri M, et al. 1995. Hemodynamic, hematological and hemorrheological evaluation of post-term pregnancy. Acta Obstet Gynecol Scand, 74:336-40.

Fugh-Berman A. 2000. Herbs and dietary supplements in the prevention and treatment of cardiovascular disease. Prev Cardiol, 3:24-32.

Mercwe LJ, Brown LG. 1986. Fetal outcome with oligohydramnios in the second trimester. Obstet Gynecol, 67:840-9.

Moher D, Schulz KF, Altman DG, et al. 2001. The revised CONSORT statement for reporting randomized trials: BMC Medical Research Methodology, 1:2doi:10.1186/1471-2288-1-2.

Moore TR, Cayle JE. 1990. The amniotic fluid index in normal human pregnancy. Am J Obstet Gynecol, 162:1168-73.

Moore TR, Longo J, Leopold G, et al. 1989. The reliability and predictive volume of an amniotic fluid scoring system in severe second trimester oligohydramnios. Obstet Gynecol, 73:739-15.

Redline RW, Pappin A. 1995. Fetal thrombotic vasculopathy: the clinical significance of extensive avascular villi. Hum Pathol, 26:80-5.
Rutherford SE, Jeffrey PP, Smith CV, et al. 1987. The four-quadrant assessment of amniotic fluid volume: an adjunct to antepartum fetal heart rate testing. Obstet Gynecol, 70:353-6.

Sarno PA, Ahn OM, Brar SH, et al. 1989. Intrapartum Doppler velocimetry, amniotic fluid volume, and fetal heart rate as predictors of subsequent fetal distress. Am J Obstet Gynecol, 161:1508-14.

Sun J, Huang SH, Tan Benny K-H, et al. 2005. Effects of purified herbal extract of salvia miltiorrhiza on ischemic rat myocardium after acute myocardial infarction. Life Science, 76:2849-60.

Xu M, Liu A, Cui Y, et al. 2007. Comparative study on HPLC fingerprints of Danshenin Xiangdan injections from different manufacturers. Chin J Nat Med, 5:120-6.

Yokozawa, T, Chung HY, Oura H, et al. 1989. Isolation of a renal functionfacilitating constituent from the Oriental drug, salviae miltiorrhizae radix. Jap J Nephrology, 31:1091-9. 